Document downloaded from:

http://hdl.handle.net/10251/63405

This paper must be cited as:

Rodríguez-Abad, I.; Klysz, G.; Martínez-Sala, RM.; Balayssac, JP.; Mene-Aparicio, J. (2016). Waterfront Depth Analysis in Hardened Concrete by Means of the Nondestructive Ground-Penetrating Radar Technique. IEEE Journal of Selected Topics in Applied Earth Observations and Remote Sensing. 9(1):91-97. doi:10.1109/JSTARS.2015.2449737.

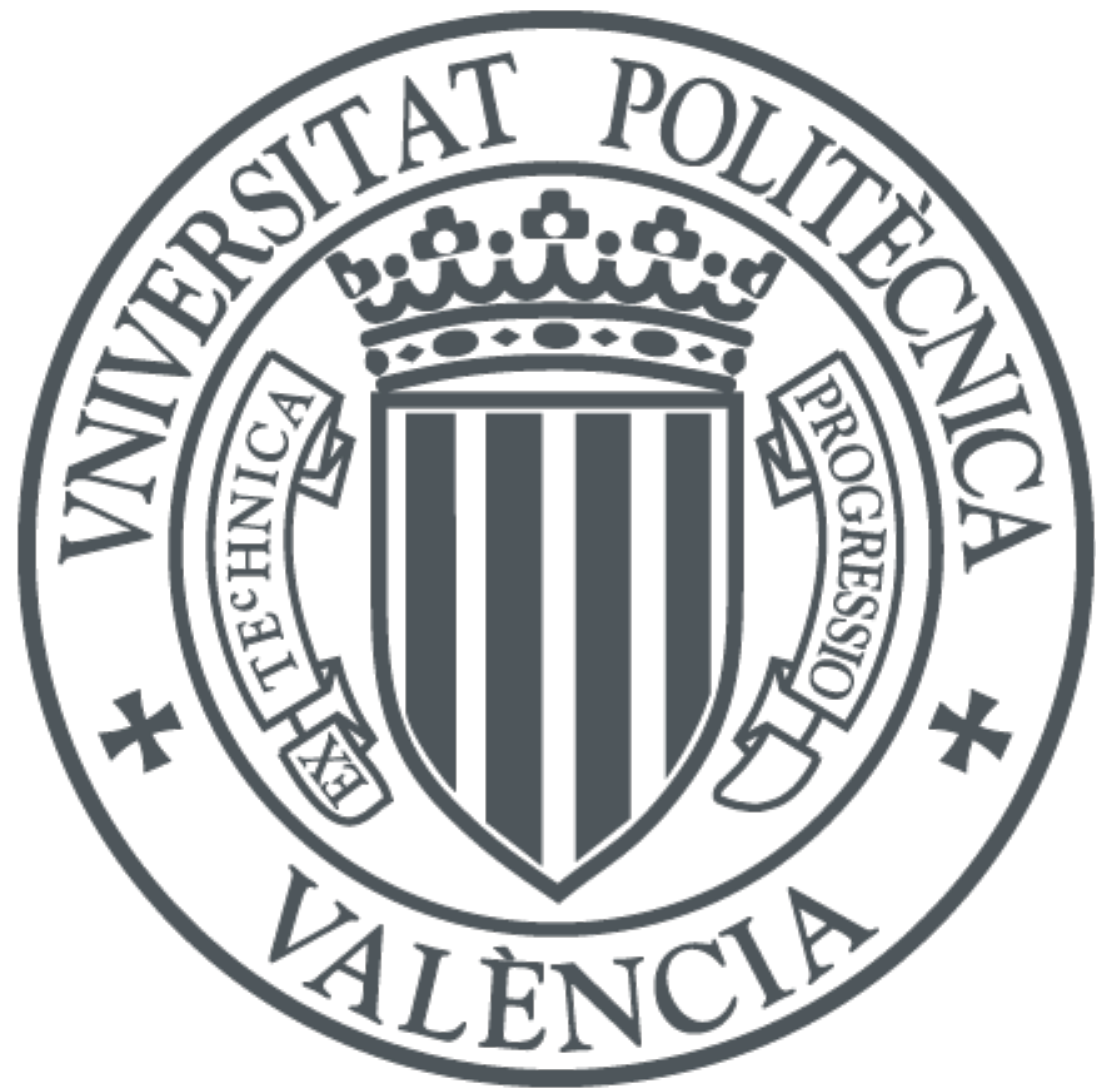

The final publication is available at

http://dx.doi.org/10.1109/JSTARS.2015.2449737

Copyright Institute of Electrical and Electronics Engineers (IEEE)

Additional Information

(C) 2015 IEEE. Personal use of this material is permitted. Permission from IEEE must be obtained for all other uses, in any current or future media, including reprinting/republishing this material for advertising or promotional purposes, creating new collective works, for resale or redistribution to servers or lists, or reuse of any copyrighted component of this work in other works. 


\title{
Waterfront depth analysis in hardened concrete by means of the non-destructive Ground-penetrating radar technique
}

\author{
Isabel Rodríguez-Abad, Gilles Klysz, Rosa Martínez-Sala, Jean Paul Balayssac, Jesús Mené-Aparicio
}

\begin{abstract}
Durability of concrete structures depends mainly on the ease whereby water and any aggressive chemical agents dissolved therein can penetrate. Therefore, measuring water penetrability in concrete structures is crucial mostly when structures are in service. In this context, non-destructive techniques play an important role. In particular, the electromagnetic waves emitted by Ground-penetrating radar (GPR) are very sensitive to the water content of the medium through which they propagate. This fact provides an interesting opportunity to analyze if the GPR technique allows the assessment of water penetrability in concrete with enough accuracy. In line with this, this paper describes the laboratory experiments and relevant analysis carried out to study the capability of GPR to assess water penetrability in hardened concrete. For this purpose, concrete specimens were fabricated and dried in an oven after 90 days of curing. They were then dipped into water and GPR measurements were taken at different intervals, based on coupling a $2.0 \mathrm{GHz}$ antenna. The results showed that the agreement between velocity increments and the waterfront advance was excellent. In addition, a specific processing of the data acquired was developed. This process included the isolation of the reflection due to the waterfront, produced just before the reflection of the bottom of the samples. As a result of this processing, the in-depth waterfront location at different times was determined with high reliability.
\end{abstract}

Index Terms - Concrete, Ground-penetrating radar, Velocity increments, Waterfront advance

\section{INTRODUCTION}

Concrete is a porous material and this implies that through the pores network aggressive substances dissolved in water can penetrate inwards. Consequently, the durability of reinforced concrete structures depends mainly on the pore structure and the level of cracking, as well as on its water content.

The authors thank the financial support for the laboratory work provided by the Universitat Politècnica de València (Spain) under the PAID-06-12 research plan through a project entitled: "Análisis de la durabilidad del hormigón por medio de la técnica no destructiva del georradar".

I. Rodríguez-Abad (isrodab@upvnet.upv.es), R. Martínez-Sala (rmsala@fis.upv.es) and J. Mené-Aparicio (jmene@mes.upv.es) are with the Universitat Politècnica de València, Camino de Vera s/n, 46022, Valencia, Spain. G. Klysz (gilles.klysz@iut-tlse3.fr) and J.P. Balayssac (balayssa@insa-toulouse.fr ) are with the Université de Toulouse; UPS, INSA; LMDC; 135, avenue de Rangueil; 31077 Toulouse Cedex 4, France.
Durability of concrete is strongly affected, among other factors, by corrosion, because it causes extensive damage and loss of structural integrity [1]. One of the main causes of its appearance is the contact of the reinforcement with water and chlorides. In low-quality concrete or exposed to aggressive environments, over time, chlorides get through the cover concrete up to the reinforcement [2].

That is why the analysis of water penetration in concrete is critical when durability studies are performed [3].

Currently, one of the procedures regulated by the European Union to provide information about the porosity of concrete is to check the degree of penetration of water under pressure. In Spain the standard that is into effect is Testing hardened concrete. Part 8: Depth of penetration of water under pressure [4].

An alternative to currently available expensive and time consuming destructive tests to determine the penetration of aggressive agents lay on nondestructive techniques, especially the Ground-penetrating radar (GPR).

GPR fundamentals are widely described by authors such as [5-6]. Commercial GPR systems generate electromagnetic waves in the microwave and radiofrequency range (from $\mathrm{MHz}$ to a few $\mathrm{GHz}$ ). The equipment consists basically of a central unit and a pair of antennas (transmitter and receiver), being the latter in a single device or separately. The control of the emission and reception of electromagnetic radiation is performed from the central unit. The transmitting antenna emits an electromagnetic pulse that travels inward a medium. Reflections will occur when the media have different dielectric constant. These reflections, that constitute the response of the material, are then collected by the receiving antenna. Furthermore, this technique can be implemented in the lab and more importantly, when the structure is in service.

Currently, some applications of GPR in the area of building and construction engineering are being studied and developed, such as: concrete moisture content assessment, determination of depths and thicknesses of foundations, location of structures or buried elements, such as vaults, underground cavities, etc. [7-9].

In particular, its application in the concrete area is providing very promising and interesting results, which highlight the strong relation between wave propagation parameters (velocity and amplitude) and concrete water content [10-12]. Some studies assess water content variation in concrete by means of the analysis of electromagnetic wave parameters [13-15]; others studies are focused on characterizing the pore 
system of hardened concrete [16] or in determining the concrete volumetric water content [17]. Even most recent studies analyze the relationship between wave parameters recorded by GPR with some indicators related to the durability, in particular, water and chloride contents [18].

However, there are few experimental studies aiming at durability control by analyzing the evolution of wave parameters while water penetrates inward in hardened concrete. Specifically, it is very interesting to study in detail the behavior of this material when a waterfront penetrates inward, using the electromagnetic field generated with a commercial GPR antenna.

Water content has a decisive influence on the dielectric properties of concrete. Therefore, changes in wave propagation will occur as a result of the advance of the waterfront and might provide reliable information, both qualitative and quantitative, about where the waterfront is located.

For all these reasons, this research focuses on the analysis of the capability of the GPR non-destructive technique for evaluating water penetration into concrete, through the assessment of the waterfront advance into the hardened concrete.

For this purpose, concrete samples were manufactured (water/cement $=0.65)$ which, after curing (90 days) and oven drying, were immersed in water. GPR measurements were performed at specific time intervals, removing the sample from water to conduct the GPR acquisition with a $2.0 \mathrm{GHz}$ center frequency antenna. Two different processing procedures were developed in order to analyze which one offered more reliable data of the waterfront advance. Firstly, propagation velocities were calculated. Secondly, a specific processing of the data was developed. This process consisted of the isolation of the reflection due to the waterfront, produced just before the reflection of the bottom of the samples. Thisprovided the determination of the waterfront location assessed at the centimeter scale at different times.

\section{EXPERIMENTAL PROGRAM}

\section{A. Samples preparation}

Experiments were conducted on ordinary concrete samples, which mixtures proportions and components main characteristics are summarized in Table I.

The tests were conducted on 24 concrete samples of dimensions $0.20 \mathrm{~m} \times 0.20 \mathrm{~m} \times 0.12 \mathrm{~m}$ without reinforcing bars. After casting, concrete samples, covered with plastic film to avoid water evaporation, were kept in the molds for 2 days. Then the samples were removed from their molds and cured by immersion in a wet chamber for a period of 28 days in order to stabilize hydration phenomenon (in accordance with the standard [19]). After this process, they were left to conduct the curing process to atmospheric ambient up to 90 days. With this age the samples were introduced in an oven $\left(105^{\circ} \mathrm{C}\right)$ to carry out their complete drying. When the mass of a sample decreased less than the $0.1 \%$, after being 24 hours in the oven, the sample was considered to be dry.
TABLE I

\begin{tabular}{|c|c|c|c|}
\hline \multicolumn{3}{|c}{ MIXTURE PROPORTIONS AND CHARACTERISTICS } \\
\hline W/C ratio & $\begin{array}{c}\text { CEM I } \\
\left(\mathbf{k g} / \mathbf{m}^{\mathbf{3}}\right)\end{array}$ & $\begin{array}{c}\text { Water } \\
\left(\mathbf{k g} / \mathbf{m}^{\mathbf{3}}\right)\end{array}$ & $\begin{array}{c}\text { Round aggregate } \\
\left(\mathbf{k g} / \mathbf{m}^{\mathbf{3}}\right)\end{array}$ \\
\hline 0.65 & 298.0 & 193.7 & 659.9 \\
\hline $\begin{array}{c}\text { Sand } \\
\left(\mathbf{k g} / \mathbf{m}^{\mathbf{3}}\right)\end{array}$ & $\begin{array}{c}\text { Compressive strength at } \\
\mathbf{2 8} \text { days }(\mathbf{M P a})\end{array}$ & \multicolumn{2}{|c|}{$\begin{array}{c}\text { Additive Sika Viscocrete } \\
\mathbf{3 4 2 5}\left(\mathbf{k g} / \mathbf{m}^{\mathbf{3}}\right)\end{array}$} \\
\hline 1225.5 & 47.9 & \multicolumn{2}{|c|}{1.8} \\
\hline
\end{tabular}

Subsequently, the samples were taken out of the oven and sealing paint was applied in all surfaces, except for the one that would be in contact with water and the opposite one. Finally, samples were immersed into $3 \mathrm{~cm}$ of water (Fig. 1a).

After GPR measurements were performed, specimens were broken in two parts and the existing waterfronts were marked and measured by visual inspection, according to the standard [4]. By means of the visual inspection, it could be observed that the distribution consisted of a saturated zone and a dry zone (Fig. 1b).
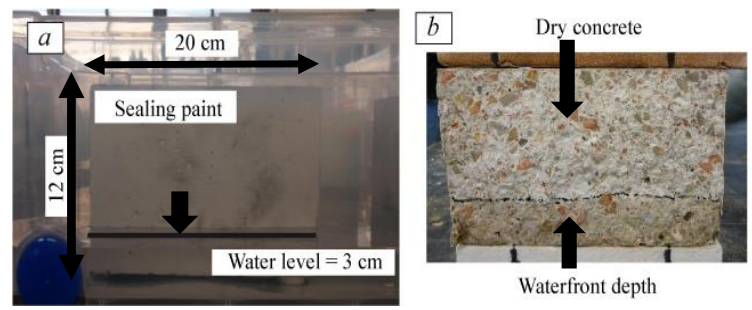

Fig. 1. (a) Concrete samples immersed into water; (b) Waterfront marked in the sample after breaking the samples in two pieces.

\section{B. GPR acquisition}

GPR measurements were carried out using a SIR-3000 system with a $2 \mathrm{GHz}$ ground coupled antenna, developed by Geophysical Survey Systems Inc. (GSSI). To minimize the border effect a squared grid mesh ( 9 acquisition points) of 10 $\mathrm{cm} \times 10 \mathrm{~cm}$ was designed (Fig. 2a). The measurements in each point were static and consisted in recording 400 scans by placing the antenna on the opposite surface to the one immersed into water. To enhance the reflected waveforms, a metallic reflector plate was placed beneath samples (Fig. 2b).

This study is focused on the analysis of the variations occurring in a electromagnetic waves, when concrete samples were at two different water content stages: after drying process and after being immersed into water for a period of time. Firstly, the survey was conducted by measuring the time delays between waves arrivals, before introducing the samples into water (calibration session). Secondly, GPR measurements were recorded on a regular basis. Every 20 minutes a sample was taken out of the water and GPR and mass measurements were conducted. Then, the samples were broken and the real waterfront depth was measured. 

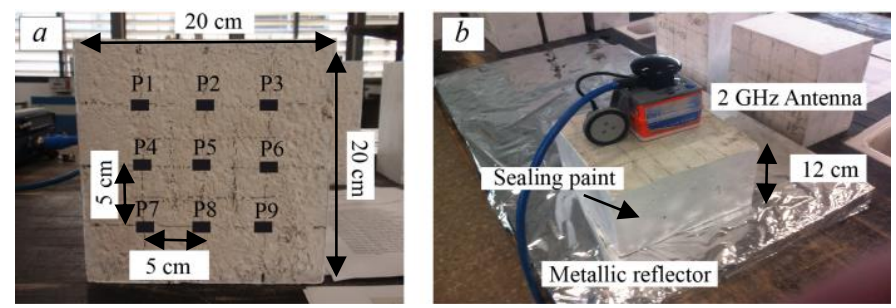

Fig. 2. (a) Location of the GPR acquisition points; (b) Static GPR acquisition.

\section{RESULTS AND DISCUSSION}

\section{A. Water content absorption parameters}

Firstly, water content coefficient $\left(C_{A}\right)$ was calculated (Table II).

$$
C_{A}(\%)=\frac{M_{i m}-M_{d}}{M_{d}} \cdot 100
$$

where $M_{d}$ is the dry mass of the sample and $M_{i m}$ is the mass after the immersion into water.

Secondly, after breaking the sample, the waterfront depth was measured in both sides of the broken sample (Fig. 1b). The final waterfront depth $\left(W_{f}\right)$ value employed to correlate with the GPR data was the average of the front line marked by visual inspection in both sides (Table II).

TABLE II

WATER CONTENT PARAMETERS

\begin{tabular}{|c|c|c|c|c|c|c|c|}
\hline Sample & $\begin{array}{c}\boldsymbol{t}_{\boldsymbol{i m}^{*}} \\
(\mathbf{m i n})\end{array}$ & $\begin{array}{c}\boldsymbol{W}_{\boldsymbol{f}} \\
(\mathbf{c m})\end{array}$ & $\begin{array}{c}\boldsymbol{C}_{\boldsymbol{A}} \\
(\boldsymbol{\%})\end{array}$ & Sample & $\begin{array}{c}\boldsymbol{t}_{\boldsymbol{i m}^{*}} \\
(\mathbf{m i n})\end{array}$ & $\begin{array}{c}\boldsymbol{W}_{\boldsymbol{f}} \\
(\mathbf{c m})\end{array}$ & $\begin{array}{c}\boldsymbol{C}_{\boldsymbol{A}} \\
(\boldsymbol{\%})\end{array}$ \\
\hline $\mathbf{1}$ & 20 & 0.52 & 0.31 & $\mathbf{1 3}$ & 260 & 3.58 & 1.33 \\
\hline $\mathbf{2}$ & 40 & 1.27 & 0.46 & $\mathbf{1 4}$ & 305 & 3.56 & 1.42 \\
\hline $\mathbf{3}$ & 60 & 1.21 & 0.50 & $\mathbf{1 5}$ & 325 & 3.65 & 1.51 \\
\hline $\mathbf{4}$ & 80 & 1.88 & 0.63 & $\mathbf{1 6}$ & 345 & 3.94 & 1.64 \\
\hline $\mathbf{5}$ & 100 & 1.97 & 0.68 & $\mathbf{1 7}$ & 365 & 4.09 & 1.64 \\
\hline $\mathbf{6}$ & 120 & 2.14 & 0.74 & $\mathbf{1 8}$ & 385 & 4.60 & 1.79 \\
\hline $\mathbf{7}$ & 140 & 2.36 & 0.82 & $\mathbf{1 9}$ & 405 & 4.22 & 1.77 \\
\hline $\mathbf{8}$ & 160 & 2.56 & 0.88 & $\mathbf{2 0}$ & 425 & 4.17 & 1.80 \\
\hline $\mathbf{9}$ & 180 & 2.71 & 0.91 & $\mathbf{2 1}$ & 445 & 4.42 & 1.80 \\
\hline $\mathbf{1 0}$ & 200 & 2.72 & 0.99 & $\mathbf{2 2}$ & 465 & 4.66 & 1.98 \\
\hline $\mathbf{1 1}$ & 220 & 2.80 & 0.98 & $\mathbf{2 3}$ & 485 & 4.56 & 2.01 \\
\hline $\mathbf{1 2}$ & 240 & 3.11 & 1.12 & $\mathbf{2 4}$ & 505 & 4.66 & 1.93 \\
\hline
\end{tabular}

\section{B. Effect of the water content on GPR records}

GPR signals were processed and analyzed using RADAN NT software (GSSI).

Fig. 3a shows schematically a typical trace of the recorded radar signals. In this trace two different waveforms can be differentiated. The energy propagated directly from the emitter to receiver in the air, along with the energy received after propagating in the outermost surface of the sample, is recorded and it is called direct wave $(D)$. The energy that is reflected by the metallic reflector located at the bottom of the sample is recorded. This second part is named reflected wave $(R)$. It is noted that the polarity of the direct wave and the reflected are reversed because of the placement of the metallic reflector. As it is indicated in Fig. 3a the direct and reflected waves consist of different peaks, maximums and minimums $(D 1, D 2 \ldots R 2, R 3)$.
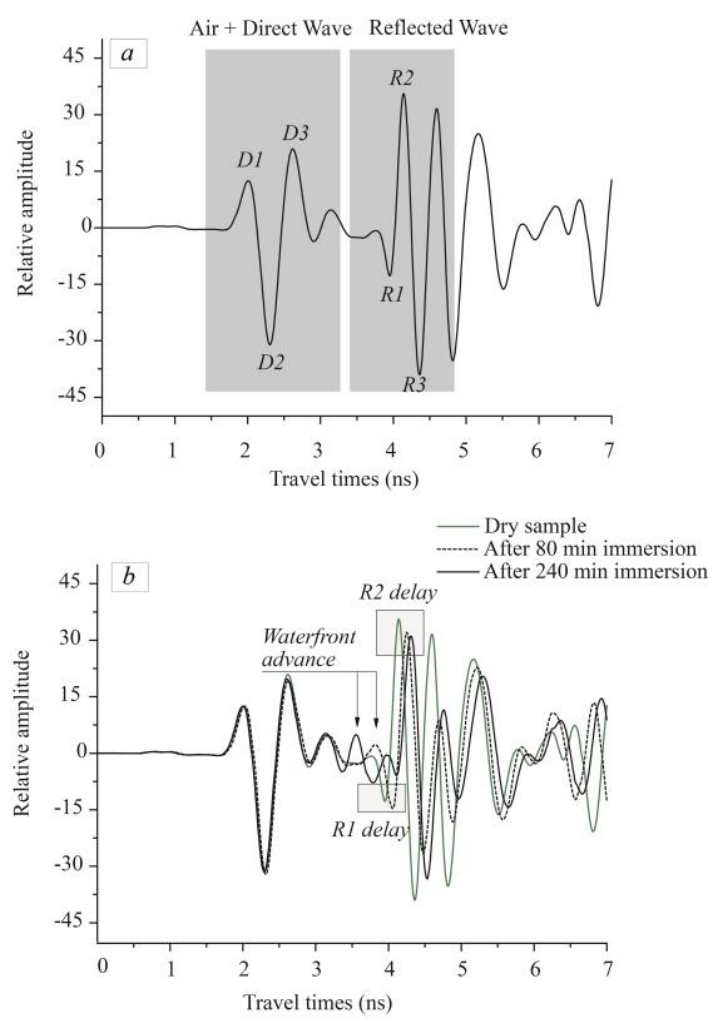

Fig. 3. (a) Typical signal recorded with the $2 \mathrm{GHz}$ antenna on a concrete slab; (b) Typical signals recorded when the sample was dry and after 80 and 240 minutes immersed into water.

As it can be observed in Fig. 3b, the presence of water in concrete did not affect the direct wave, since the wave travelled by the dry part of the concrete sample. Unlike the direct wave, the reflected waves recorded after immersion were altered due to the presence of water. In the signals registered when samples had been in water less than 120 minutes, it can be observed that the reflection in the interface between the dry concrete and wet concrete and the reflection at the bottom of the sample were overlapped. From that moment until the end of the experiment these two reflections were separated enough, being in these cases easier to be identified. However, regardless of the moment of the waterfront location, a general pattern in the reflected wave at the bottom of the sample was observed. Arrival times were delayed and the amplitude peaks were smaller.

\section{Effect of the water immersion on wave velocities}

Arrival times of the direct and reflected waves were measured in the acquired traces when the samples were dry and when they were immersed into water for a period of time. By means of the difference in arrival times between the direct wave and the reflected wave $\left(\Delta t_{R}^{D}\right)$ propagation wave velocities $(v)$ were calculated according to (2):

$$
v=\frac{2 \cdot d}{\Delta t_{D}^{R}}=\frac{\left(2 \cdot \sqrt{h^{2}+\left(\frac{d_{0}}{2}\right)^{2}}\right)}{\Delta t_{D}^{R}}
$$

where $d$ is the semi-distance that travelled the reflected wave, $d_{0}$ is the distance between emitter and receiver $(4 \mathrm{~cm})$ and $h$ is the thickness of the medium. 
The objective of this analysis is to assess the waterfront effect on wave parameters without knowing its location.

It is important to highlight that the calculated velocity depends on the contribution of two velocities: the velocity in the dry and in the wet concrete.

The electrical properties of concrete are greatly affected when the water content increases. In fact, an increase of water content results in an increase in polarization and hence the value of the propagation velocity decreases $(v)$. However, when analyzing the records it is difficult to assess the exact arrival time of each wave, making it difficult to calculate the propagation velocity.

As some authors pointed out [20-21], it is complex to establish which one of the peaks that are comprised in a wavelet is representative of the exact wave arrival time.. In the case of the direct wave an overlap occurs between the air wave (between emitter and receiver) and direct wave itself. It is very complex to establish which peak indicates the arrival of the direct wave. As for the reflected wave, when it travels through the medium suffers attenuation. Therefore, it is also very complex to estimate which one is the best representative of its arrival.

For all these reasons, in this work velocities were calculated with all possible combinations of time intervals between peaks of the direct wave $(D 1, D 2$ and $D 3)$ and the reflected wave $(R 1, R 2$ and $R 3$ ) (Fig. 3a). Then we analyzed which combination provided more accurate information regarding the waterfront advance. For each sample, the arrival times of the 9 GPR acquisition points (Fig. 2a) were calculated averaged and from them and by means of (2) the propagation velocities for each sample were calculated. This procedure was followed when the samples were dry and after being immersed into water.

Finally, velocity increments that occurred when the samples were dry and after immersion were obtained for each sample:

$$
\Delta v\left[\frac{c m}{n s}\right]=v_{i m}-v_{d}(3)
$$

where $v_{d}$ is the velocity when the sample is dry and $v_{i m}$ when is immersed into water. The values of velocity increments versus waterfront advance $\left(W_{f}\right)$ are depicted in Fig. 4.
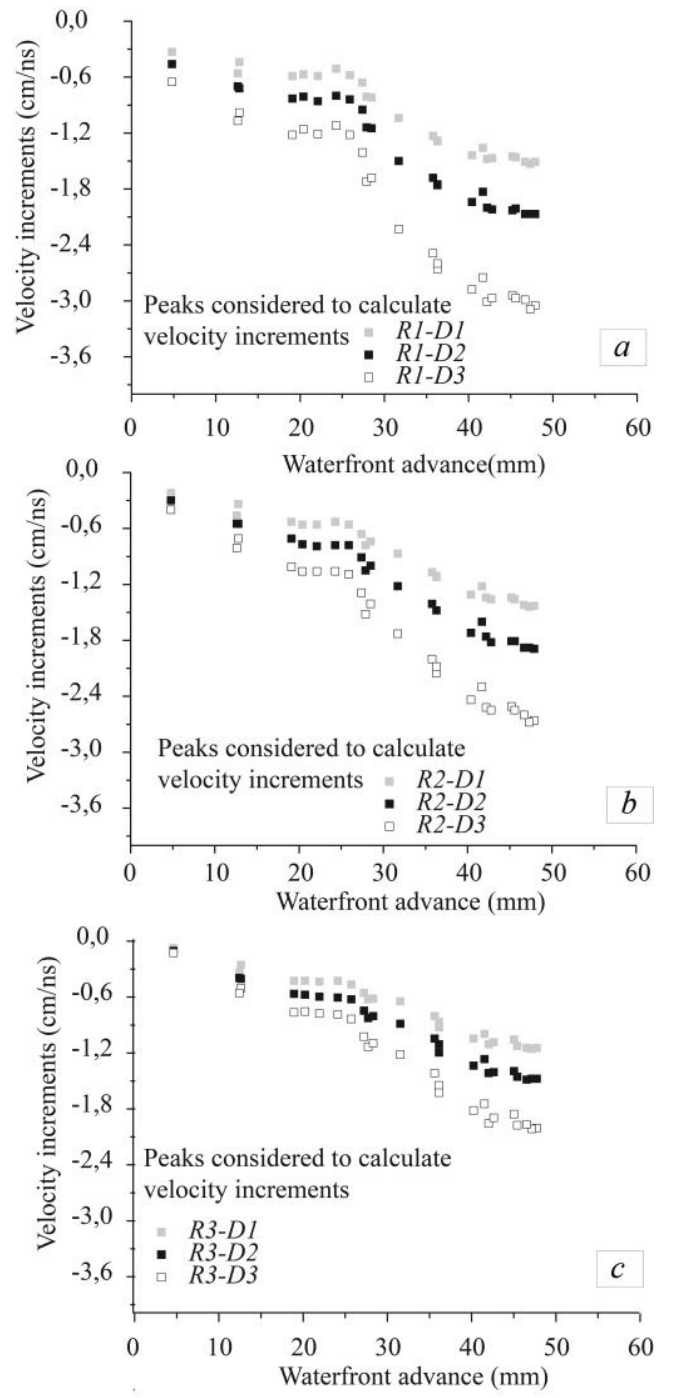

Fig. 4. Velocity increments $(\mathrm{cm} / \mathrm{ns})$ versus waterfront advance determined after breaking the samples. Velocity increments showed the same trend regardless of the peak used to calculate velocities. That is, velocity increments were always negative since, as mentioned, the propagation velocities of the waves fell after the samples were immersed in water.

The equations of the curves that provided a better fit between velocity increments and the waterfront advance were calculated (Table III).

TABLE III

EQUATIONS OF THE ADJUSTMENTS BETWEEN VELOCITY INCREMENTS (CM/NS) AND WATERFRONT ADVANCE (CM).

\begin{tabular}{|c|c|c|c|c|c|c|c|}
\hline \multirow{3}{*}{$\begin{array}{l}\text { Peaks considered } \\
\text { to calculate } \\
\text { velocity increments }\end{array}$} & \multicolumn{7}{|c|}{$\begin{array}{c}2^{\text {nd }} \text { Order Polynomial Adjustment } \\
y(x)=a+b x+c x^{2}\end{array}$} \\
\hline & \multicolumn{2}{|c|}{$a$} & \multicolumn{2}{|c|}{$b$} & \multicolumn{2}{|c|}{$c$} & \multirow[t]{2}{*}{$R^{2}$} \\
\hline & Value & $\sigma^{*}$ & Value & $\sigma^{*}$ & Value & $\sigma^{*}$ & \\
\hline$R 1-D 1$ & -0.24 & 0.13 & -0.009 & 0.009 & -0.001 & 0.0015 & 0.93 \\
\hline$R 1-D 2$ & -0.33 & 0.15 & -0.02 & 0.010 & -0.005 & 0.0020 & 0.94 \\
\hline RI-D3 & -0.42 & 0.25 & -0.03 & 0.020 & -0.007 & 0.0030 & 0.93 \\
\hline$R 2-D 1$ & -0.15 & 0.08 & -0.01 & 0.006 & -0.003 & 0.0001 & 0.97 \\
\hline$R 2-D 2$ & -0.19 & 0.09 & -0.02 & 0.006 & -0.004 & 0.0001 & 0.98 \\
\hline R2-D3 & -0.22 & 0.14 & -0.03 & 0.01 & -0.001 & 0.0002 & 0.97 \\
\hline R3-DI & -0.04 & 0.06 & -0.02 & 0.005 & -0.002 & 0.0001 & 0.97 \\
\hline R3-D2 & -0.05 & 0.07 & -0.02 & 0.005 & -0.002 & 0.0001 & 0.98 \\
\hline R3-D3 & -0.02 & 0.10 & -0.03 & 0.007 & -0.002 & 0.0001 & 0.97 \\
\hline
\end{tabular}


The behavior of the velocity increments as a function of the waterfront advance were very well described by the curves shown in Table III. This fact is pointed out by the high determination coefficient values found $\left(R^{2} \geq 0,93\right)$, regardless of the peaks considered to calculate the velocity increment. Despite of the fact that with any of the three peaks $(R 1, R 2$ and $R 3)$ the results are statistically very similar, it is interesting to notice that when the $R 1$ peak was not considered the values of that coefficient $\left(R^{2}\right)$ were especially high $(0,97$ and 0,98$)$. This is quite important, since $R l$ is a peak that not always is easily identifiable. This might be explained because, as it is mentioned above, in the samples that were in water less than 120 minutes the reflections occurred in two interfaces (dry and wet concrete and wet concrete and metallic reflector) were overlapped (Fig. 3b), and the overlap jammed basically $R I$ peak.

\section{Waterfront advance assessment}

The reflected wave was affected by the water content. Nevertheless, in the time interval named as reflected wave $(R)$, when the samples were immersed into water, two reflections occurred due to two interfaces (Fig. 5).

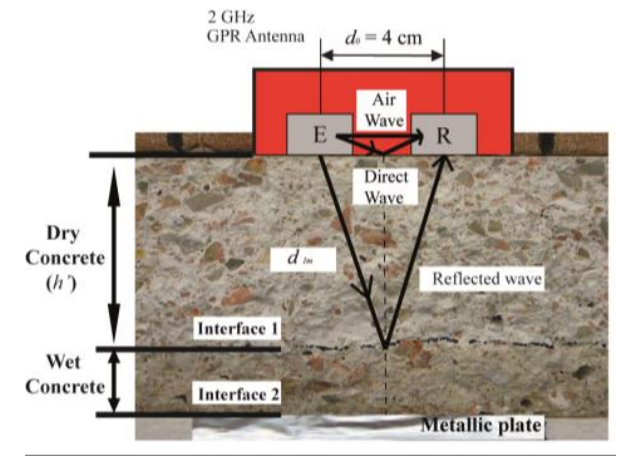

Fig. 5. Paths of the electromagnetic rays when the antenna was placed on the immersed samples.

Interface 1, was the one due to the dielectric contrast between the dry and wet concrete. Interface 2, was due to the dielectric contrast between the wet concrete and the metallic reflector. But, as this occurred in a very short period of time, both reflections happened to be overlapped, at least for the measurement acquired for the first 120 minutes after immersion (Fig. 6a). In the following acquisitions Interface 1 and 2 could be identified separately (Fig. 6b).
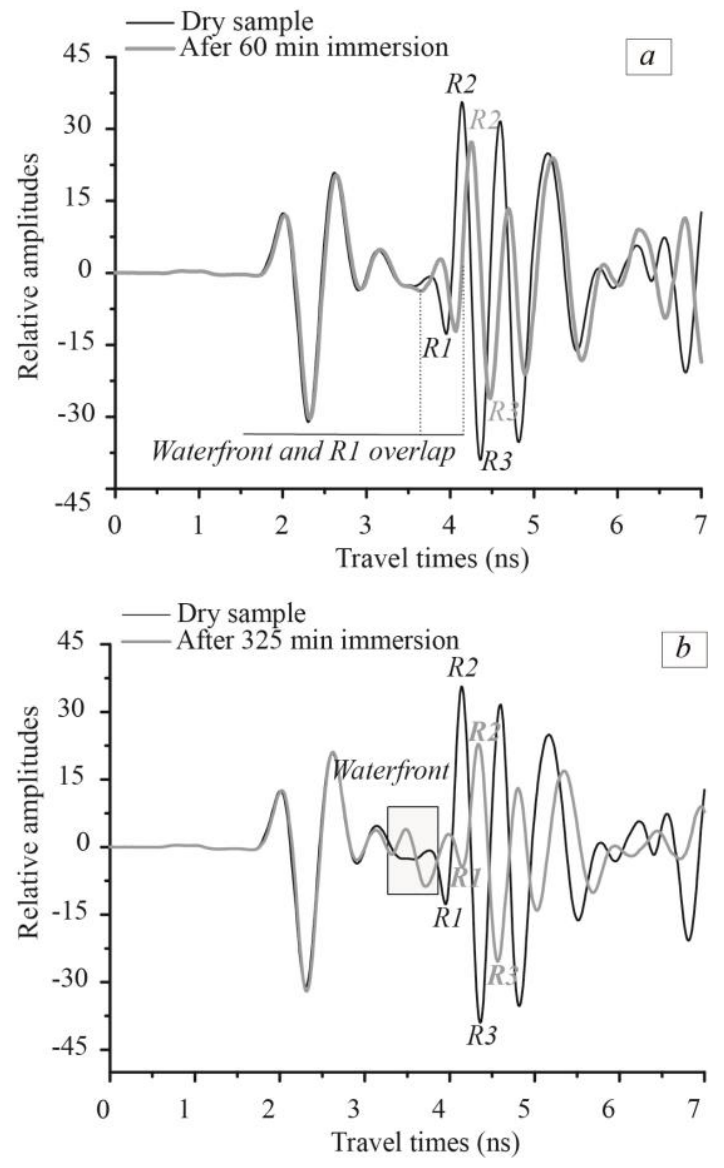

Fig. 6. Typical radar signals before 120 minutes of immersion into water (a) and after 120 minutes of immersion (b).

When the sample was immersed into water for $120 \mathrm{~min}$ the waterfront advanced $2.14 \mathrm{~cm}$ according to the visual inspection (Table II). In this sample the waves velocity in dry concrete ranged from $10.13 \mathrm{~cm} / \mathrm{ns}$ to $18.00 \mathrm{~cm} / \mathrm{ns}$, depending on the peak considered to be calculated. These velocities correspond to a wave length from $5.07 \mathrm{~cm}$ to $9.00 \mathrm{~cm}$ (Table IV).

TABLE IV

VELOCITIES AND WAVELENGTHS IN DRY CONCRETE WHEN THE SAMPLE WAS IMMERSED INTO WATER FOR 120 MINUTES.

\begin{tabular}{|c|c|c|c|c|c|c|c|c|c|}
\hline & $\begin{array}{l}R 1 \\
D 1\end{array}$ & $\begin{array}{l}R 1 \\
D 2\end{array}$ & $\begin{array}{l}R 1 \\
D 3\end{array}$ & $\begin{array}{l}R 2 \\
D 1\end{array}$ & $\begin{array}{l}R 2 \\
D 2\end{array}$ & $\begin{array}{l}R 2 \\
D 3\end{array}$ & $\begin{array}{l}R 3 \\
D 1\end{array}$ & $\begin{array}{l}R 3 \\
D 2\end{array}$ & $\begin{array}{l}R 3 \\
D 3\end{array}$ \\
\hline $\begin{array}{c}v \\
(c m / n s)\end{array}$ & 12.5 & 14.7 & 18.0 & 11.3 & 13.1 & 15.7 & 10.1 & 11.6 & 13.5 \\
\hline$\lambda(\mathrm{cm})$ & 6.2 & 7.3 & 9.0 & 5.7 & 6.6 & 7.9 & 5.1 & 5.8 & 6.7 \\
\hline
\end{tabular}

Regardless of the occurrence of the overlap between Interface 1 and 2, it was necessary to define a procedure, by which the waterfront reflection (Interface 1) could be identified. In order to isolate the reflection due to the waterfront interface (Interface 1), the following processing procedure was developed. The aim of this procedure was to be able to subtract two signals: the one acquired when the sample was dry and the same trace, but acquired after a time of immersion. Firstly, wave velocities when the samples were dry $\left(v_{d r y}\right)$ were calculated(4): 


$$
v_{d r y}=\frac{2 \cdot d_{d r y}-d_{0}}{\Delta t_{D 2}^{R 2}}=\frac{\left(2 \cdot \sqrt{h^{2}+\left(\frac{d_{0}}{2}\right)^{2}}\right)-d_{0}}{\Delta t_{D 2}^{R 2}}
$$

where $h$ is the thickness of the sample, $d_{0}$ is the distance between emitter and receiver and $\Delta t_{D 2}^{R 2}$ is the time elapsed between the arrival of the direct wave (peak D2) and the reflected wave (peak $R 2$ ).

Prior to subtract the dry signal to the immersed one, it was necessary to align the dry signal with the immersed one, creating a new one named dry-corrected signal. This drycorrected signal was made of two parts. The first part remains equal to the dry signal, since the direct wave was not altered after immersion. But the second part of the dry signal had to be corrected because the reflected wave registered when the sample was dry went ahead of the reflected wave registered when the sample had been in water. Then both signals (drycorrected and immersed signal) were aligned taking as a reference $R 3$ of the immersed signal (Fig. 3a). It was chosen this peak as a reference to perform the alignment, because it was considered that this peak should not be affected or overlapped with the reflection of Interface 1. Finally, both signals, dry-corrected and immersed into water, were in condition to be subtracted. As a result of the subtraction the reflection of the waterfront could be identified (Fig. 7).

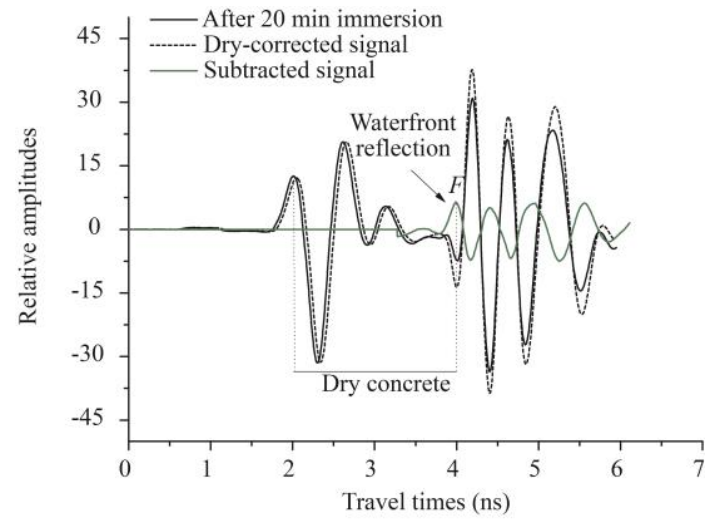

Fig. 7. Waterfront reflection isolation by signals subtraction.

Once the dry velocity was calculated and the reflection due to the waterfront (Interface 1) identified, the thickness of the waterfront $\left(W_{f}\right)$ can be determined by means of simple geometry.

$$
\begin{gathered}
d_{I m}=\frac{\left(\Delta t_{D 2}^{F} \cdot v_{d r y}\right)+d_{0}}{2} \\
h^{\prime}=\sqrt{d_{I m}^{2}-\left(\frac{d_{0}}{2}\right)^{2}}(6) \\
W_{f}=h-h^{\prime}(7)
\end{gathered}
$$

where $d_{i m}$ is the path travelled from emitter to waterfront interface, $\Delta t_{D 2}^{F}$ is the two-way travel time between the reflection of the waterfront (Interface 1) and the $2^{\text {nd }}$ peak of the direct wave (D2) and $h^{\prime}$ is the dry concrete thickness. The final $W_{f}$ calculated from GPR data are detailed in Table V.
TABLE V

\begin{tabular}{|c|c|c|c|c|c|}
\multicolumn{7}{|c|}{ WATERFRONT ADVANCE PARAMETERS } \\
\hline Sample & $\begin{array}{c}\boldsymbol{W}_{\boldsymbol{f}} \\
(\boldsymbol{G P R})\end{array}$ & $\begin{array}{c}\boldsymbol{W}_{\boldsymbol{f}} \\
\text { (Visual } \\
\text { inspection) }\end{array}$ & Sample & $\begin{array}{c}\boldsymbol{W}_{\boldsymbol{f}} \\
(\boldsymbol{G P R})\end{array}$ & $\begin{array}{c}\boldsymbol{W}_{\boldsymbol{f}} \\
\text { (Visual } \\
\text { inspection })\end{array}$ \\
\hline $\mathbf{1}$ & 0.90 & 0.52 & 13 & 3.63 & 3.58 \\
\hline $\mathbf{2}$ & 1.29 & 1.27 & 14 & 3.90 & 3.56 \\
\hline $\mathbf{3}$ & 1.54 & 1.21 & 15 & 3.75 & 3.65 \\
\hline $\mathbf{4}$ & 1.76 & 1.88 & 16 & 4.19 & 3.94 \\
\hline $\mathbf{5}$ & 1.89 & 1.97 & 17 & 4.25 & 4.09 \\
\hline $\mathbf{6}$ & 2.23 & 2.14 & 18 & 4.71 & 4.60 \\
\hline $\mathbf{7}$ & 2.31 & 2.36 & 19 & 4.44 & 4.22 \\
\hline $\mathbf{8}$ & 2.47 & 2.56 & 20 & 4.42 & 4.17 \\
\hline $\mathbf{9}$ & 2.81 & 2.71 & 21 & 4.70 & 4.42 \\
\hline $\mathbf{1 0}$ & 2.91 & 2.72 & 22 & 4.87 & 4.66 \\
\hline $\mathbf{1 1}$ & 2.89 & 2.80 & 23 & 4.78 & 4.56 \\
\hline $\mathbf{1 2}$ & 3.21 & 3.11 & 24 & 4.80 & 4.66 \\
\hline
\end{tabular}

The difference of the waterfront advance calculated by means of GPR and the one derived from the visual inspection of the broken samples was very small. The maximum difference was found in sample 1 (after 20 minutes immersion). This result was expected, since it was difficult to assess visually the waterfront thickness in the first session, due to several factors: the small amount of water content increase, the heterogeneity of the concrete surface and the quick evaporation of water in the analyzed surface, limiting the identification of the separation of wet and dry concrete.

The waterfront advance obtained by visual inspection and by processing GPR data was fitted using the first values as the independent variable and second as the dependent variable (Fig. 8).

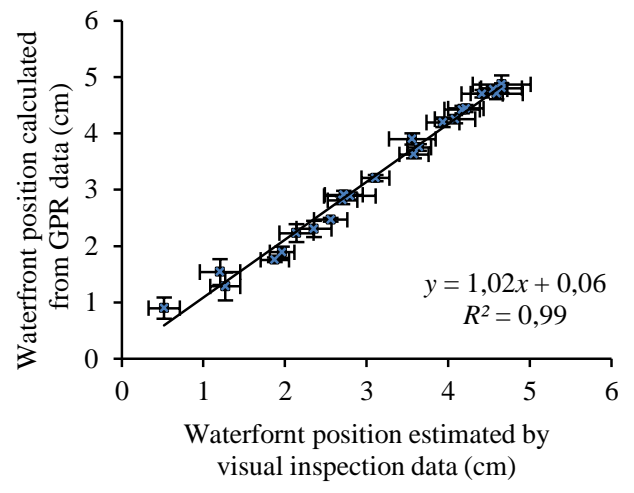

Fig. 8. Adjustment between waterfront advance obtained by means of the GPR data and visual inspection data after breaking the samples.

The result of the adjustment was very interesting, since the curve resulting from the adjustment was almost $y=x$. That is, a curve with a slope of 1 , and centered in the origin. Besides, this great agreement was supported by a determination coefficient of $R^{2}=0,99$, validating the high accuracy of the waterfront determination calculated from GPR data. In addition, the average standard deviation of the waterfront determination by means of GPR was slightly smaller $(\sigma=0,10$ $\mathrm{cm})$ than when it was determined by visual inspection $(\sigma=$ $0,22 \mathrm{~cm}$ ). 


\section{CONCLUSIONS}

The results of the experimental study presented in this paper are a proof of the great capability of the GPR technique, using a $2 \mathrm{GHz}$ commercial antenna, to carry out nondestructive testing of the water penetration in hardened concrete. The relevance of this fact is evident when taking into account the close link between the vulnerability of reinforced concrete structures and the property that indicates how easily penetrate far inside the water and aggressive agents bearing dissolved chemicals.

Two different methodologies have been evaluated to assess the waterfront thickness from GPR measurements and in both cases the results are of great interest. The changes occurred in GPR signals recorded on samples when they had been immersed in water during different time intervals were analyzed. In particular, it was found that the velocity increment described with great approximation the variation of the waterfront advance, regardless of the peaks considered to calculate the propagation velocity and without taking into account when the waterfront reflection occurred. This result was confirmed by the high determination coefficients that were obtained when the variables were fitted.

Besides, a signal processing procedure to isolate the reflection that took place in the waterfront was successfully developed. Once this reflection was identified, it was possible to determine the waterfront advance. The agreement between the values found with those obtained visually after breaking the samples pointed out the goodness of the method.

However, further research will be needed with a larger number of samples, of dimensions, different water / cements ratios and types of concrete (included reinforced concrete) to check the generality of these results and determine the range of water penetration for which the procedure is valid.

\section{ACKNOWLEDGMENT}

The authors thank the Laboratorio de Materiales de Construción of the Escuela Técnica Superior de Ingeniería de la Edificación de la Universitat Politècnica de València technical team for the valuable collaboration.

\section{REFERENCES}

[1] P. Schiessel, "Corrosion of steel in concrete". RILEM report Technical Committee 60-CSC. Chapman \& Hall, London., 1998

[2] A. M. Neville, "Maintenance and durability of structures," Concrete International, pp. 52-56, 1997

[3] M. B. Otieno, M. G. Alexander. and H. D. Beushausen, "Corrosion in cracked and uncracked concrete - influence of crack width, concrete quality and crack reopening", Magazine of Concrete Research, vol. 62, 6, pp. 393-404, 2010.

[4] Testing hardened concrete. Part 8: Depth of penetration of water under pressure. UNE-EN 12390-8, 2009

[5] D. Daniels, "Ground-Penetration radar". The Institution of Electrical Engineers, London, UK, 2004.

[6] Annan A.P., "Ground Penetrating Radar: Principles, Procedures and Applications". Sensors And Software Inc. Mississauga, 2003.

[7] I Rodríguez-Abad, R. Martínez-Sala, R. Capuz, R. Diez Barra, F. García García, "Assessment of the variation of the moisture content in the Pinus pinaster Ait using the nondestructive GPR technique", Materiales de Construcción, vol. 61, no. 301, pp. 143-156, 2011.

[8] V. Pérez, F. García and I. Rodríguez-Abad, "GPR evaluation of the damage found in the reinforced concrete base of a block of flats: A case study", NDT \& E International, vol. 41, no. 5, pp. 341-353, 2008.
[9] V. Pérez, "Innovative inspection procedures for effective GPR surveying of buildings", COST ACTION TU1208-Civil engineering applications of ground penetrating radar. Working Group Progress Meeting, 2014.

[10] R. Martínez-Sala, I. Rodríguez-Abad and I. Del Val, "Effect of penetration of water under pressure in hardened concrete on GPR signals", Proceedings of the International Workshop on Advanced Ground Penetrating Radar (IWAGPR), Nantes, France, 2013.

[11] I. Rodríguez-Abad, R. Martínez-Sala, J. Mené, G. Klysz, " Water penetrability in hardened concrete by GPR", Proceedings of the 15th International Conference on Ground Penetrating Radar; Brussels, 2014.

[12] F. Tosti and L. Bianchini, "Determination, by using GPR, of the volumetric water content in structures, substructures, foundations and soil", COST Action TU 1208. Civil Engineering applications of Ground Penetrating Radar. First General Meeting- Proceedings, 2013.

[13] Z. M. Sbartai, S. Laurens, J. P. Balayssac, G. Arliguie and G. Ballivy, "Ability of the direct wave of radar ground-coupled antenna for NDT of concrete structures", NDT\&E International, vol.39, no. 5, pp. 400-407, 2006

[14] S. Laurens, J. P. Balayssac, J. Rhazi,, G. Klysz, and G. Arliguie, "Nondestructive evaluation of concrete moisture by GPR: experimental study and direct modeling", Materials and Structures, vol. 38, no. 283, pp. 827-832, 2005.

[15] Z. M. Sbartai, S. Laurens, J. P. Balayssac, G. Ballivy and G. Arliguie, "Effect of concrete moisture on radar signal amplitude", ACI Materials Journal, vol. 103, no. 6, pp. 419- 426, 2006.

[16] W. L. Lai and W. F. Tsang, W.F., "Characterization of pore systems of air/water-cured concrete using ground penetrating radar (GPR) through continuous water injection", Construction and Building Materials, vol. 22, pp. 250-256, 2008.

[17] W. L. Lai, S. C. Kou, W. F. Tsang and C.S. Poon, "Characterization of concrete properties from dielectric properties using ground penetrating radar", Cement and Concrete Research, vol. 39, pp. 687-695, 2009.

[18] G. Villain, X. Derobert, M. Sbartai and J.P. Balayssac, "Evaluation of concrete water content and other durability indicators by electromagnetic measurements", Proceedings of the 13th International Conference on Ground Penetrating Radar, Lecce, Italy, 2010.

[19] Testing hardened concrete. Part 2: Making and curing specimens for strength test. UNE-EN 12390-2:2009

[20] R. Yelf and D. Yelf D, "Where is the True Time Zero?", Electromagnetic Phenomena, vol. 7, no. 1, pp. 158-163, 2006.

[21] R. Martínez-Sala, I. Rodríguez-Abad and J. Tapia, "Analysis of the reflected wave arrival position in timber specimens emitted by GPR with an antenna of 1,6 GHz", Proceedings of the $2^{\text {nd }}$ International Conference on Construction and Building Research, Valencia, Spain, 2012. 